\title{
Predictive value of resistive index in graft survival after kidney transplant
}

\author{
Saverio Forte, Pasquale Martino, Silvano Palazzo, Matteo Matera, Floriana Giangrande, \\ Francesco Paolo Selvaggi, Pasquale Ditonno, Michele Battaglia \\ Urology, Andrology and Kidney Transplantation Unit, Department of Emergency and Organ Transplantation, \\ University of Bari, Bari, Italy.
}

\begin{abstract}
Summary Introduction: The intrarenal resistance index (RI) is a calculated parameter for the assessment of the status of the graft during the follow-up ultrasound of the transplanted kidney. Currently it is still unclear the predictive value of RI, also in function of the time.

Materials and Methods: We retrospectively investigated the correlation between the RI and the graft survival (GS) and the overall survival (OS) after transplantation. We evaluated 268 patients transplanted between 2003 and 2011, the mean followup was 73 months (12-136). The RI was evaluated at 8 days, 6 months, 1 year and 3 years. The ROC analysis was used to calculate the predictive value of RI and the Kaplan Mayer curves was used to evaluated the OS and PS.

Results: The ROC analysis, correlated to the GS, identified a value of RI equal to 0.75 as a cut-off. All patients was stratified according to the RI at 8 days ( $R I \leq 0,75: 212$ vs $R I>0.75: 56)$, at 6 months (RI $\leq 0.75: 237$ vs $R I>0.75: 31)$, at 1 year $(R I \leq 0.75$ : 229 vs $R I>0.75: 39)$ and at 3 years $(R I \leq 0.75: 224$ vs $R I>0.75$ 44). The RI showed statistically significant differences between the two groups in favor of those who had an RI $\leq 0.75$ only at 8 days and at 6 moths ( $p=0.0078$ and $p=0.02$ to 8 days to 6 months) on the GS. On the contrary, we observed that the RI estimated at 1 year and 3 years has not correlated with the GS. The same RI cut-off was correlate with PS after transplantation. We observed that there are no correlations between the $R I$ and $O S$.

Conclusions: The RI proved to be a good prognostic factor on survival organ when it was evaluated in the first months of follow-up after transplantation. This parameter does not appear, however, correlate with OS of the transplanted subject.
\end{abstract}

KEY WORDS: Kidney transplant; Doppler sonography; Resistive index; Graft survival; Patient survival.

Submitted 3 October 2014; Accepted 31 October 2014

\section{INTRODUCTION}

Kidney transplantation (KTx) is the optimal method of treatment in patients with end-stage kidney disease since the 1960s, and it has good outcomes in terms of morbidity, mortality and quality of life $(1,2)$. In many renaltransplantation centers, measurement of the intrarenal resistive index (RI) by means of Doppler ultrasonography is routinely used to evaluate renal allografts $(3,4)$. The RI is derived from the pulsatile flow-velocity waveform. The RI Pourcelot is a semiquantitative parameter, it is calculated on the curve speed/time as the ratio (SD)/S, where $S$ is the peak systolic velocity and $D$ the peak diastolic velocity (5). Previous cross-sectional study linked an increased intrarenal resistive index after kidney transplantation with an increased risk of graft loss or recipient death (6).

The role of RI on the Graft Survival (GS) and overall survival (OS) is still controversial, the RI appears to be related to the GS and OS; but his real role in the follow-up the patient is still not clear. According to some recent studies the RI, routinely measured at predefined time points after transplantation, reflects characteristics of the recipient but not those of the graft (7-8).

The purpose of this study was to analyze the impact of RI value on graft survival in relationship to the time of the Doppler.

\section{MATERIAL AND METHODS}

We retrospectively investigated the correlation between the RI and the GS and the OS after transplantation. We evaluated 268 patients transplanted between 2003 and 2011, the mean follow-up was 73 months (12-136). The RI was evaluated at 8 days, 6 months, 1 year and 3 years. The ROC analysis was used to calculate the predictive value of RI and the Kaplan Mayer curves was used to evaluated the OS and PS.

The RI were measured by duplex ultrasound, the Doppler spectra were obtained from the segmental arteries at three different location and the values were obtained from the mean of the three different location. The RI were measured at 8 days, 6 months, 1 year and 3 years after kidney transplantation.

The donor variables collected from the database were: age, body mass index (BMI), serum creatinine levels, clearance at the time of death and biopsy by the Remuzzi-Karpinsky score. Recipient variables were: age, BMI, time on dialysis, cold ischemia time, delayed renal function incidence, hospitalization, incidence of acute graft rejection, GS and OS.

The ROC analysis, correlated to the GS, was used to

No conflict of interest declared. 
Table 1. Diagnostic findings of cases series.

\begin{tabular}{|c|c|c|c|c|c|c|}
\hline \multicolumn{4}{|c|}{ Groups $\mathbf{R I}$ at $\mathbf{8}$ days } & \multicolumn{3}{|c|}{ Groups RI at 6 months } \\
\hline Donors & $\mathbf{R I} \leq 0,75$ & $\mathrm{RI}>0.75$ & $\mathbf{P}$ & $\mathbf{R I} \leq 0,75$ & $\mathrm{RI}>0.75$ & $\mathbf{P}$ \\
\hline Means age & $44,7 \pm 17,1(13-77)$ & $49,8 \pm 15(17-72)$ & NS & $44,9 \pm 16,9(13-77)$ & $55,7 \pm 13,4(25-75)$ & 0,001 \\
\hline Karpinsky score & $1,38 \pm 1,47(0-6)$ & $2,1 \pm 1,5(0-5)$ & 0,009 & $1,62 \pm 1,58(0-6)$ & $2,15 \pm 1,3(0-4)$ & NS \\
\hline Mean cold ischemia & $14,8 \pm 5,2(7-32)$ & $15,8 \pm 4,1(8-24)$ & NS & $15,1 \pm 4,9(7-31)$ & $19,1 \pm 6,1(12-32)$ & 0,004 \\
\hline \multicolumn{7}{|l|}{ Recipients } \\
\hline Mean age (years) & $43,5 \pm 10,6(14-65)$ & $49,5 \pm 9,1(25-62)$ & $<0,001$ & $44,8 \pm 10,2(14-64)$ & $53,2 \pm 7,5(36-65)$ & $<0,001$ \\
\hline Mean BMI (Kg/m²) & $23,7 \pm 4,5(17,3-37)$ & $22,53 \pm 3,9(16,1-31,6)$ & NS & $23,4 \pm 4,2(17,3-37,2)$ & $22,9 \pm 4,5(16,1-35)$ & NS \\
\hline Mean time of dyalisis & $67,4 \pm 54,8(3-264)$ & $101,5 \pm 68,9(3,4-339,6)$ & $<0,001$ & $72,2 \pm 55,9(3-267)$ & $82,8 \pm 70,7$ (11-339) & NS \\
\hline $\begin{array}{l}\mathrm{sCr}(\mathrm{mg} / \mathrm{dl}) \\
\text { at moment of RI }\end{array}$ & $1,77 \pm 0,70(0,7-4,0)$ & $2,5 \pm 1,17(0,9-5,7)$ & $<0,001$ & $1,60 \pm 0,50(0,8-3,5)$ & $1,78 \pm 0,52(0,9-3)$ & NS \\
\hline
\end{tabular}

identify the RI cut-off, the GS and OS were calculated by Kaplan Mayer analyses. The lang-rank test was used to compare survival curves; $p<0,05$ was considered statistically significant. Statistical analyses were performed by MedCalc software.

\section{RESULTS}

In our transplant center, 268 kidney transplant from cadaveric donors were performed. Mean follow-up time of 73 months (CI 12-136). Table 1 shows the main characteristics of donor and recipients according to RI groups.

The ROC analyze was used to identify the RI cut-off. We correlated the value of RI at 8 days, 6 months, 1 year and 3 years after kidney transplantation correlated to the GS. The area under the curve $(\mathrm{AUC}=0,647)$ was better when the RI was calculated at 8 days.

The cut-off identified was 0,75 with better relationship between sensitivity and specificity (Sensitivity: 49\%, Specificity: 82\%, p = 0,0014) (Figure 1).

This value was used as cut-off; thus all patients was stratified according to the RI at 8 days (RI $\leq 0,75$ : 212 vs RI >0.75: 56), at 6 months (RI $\leq 0,75: 237$

\section{Figure 1.}

The ROC analyze identified as cut-off 0,75 with better relationship between the sensitivity and specificity $(A \cup C=0,647)$.

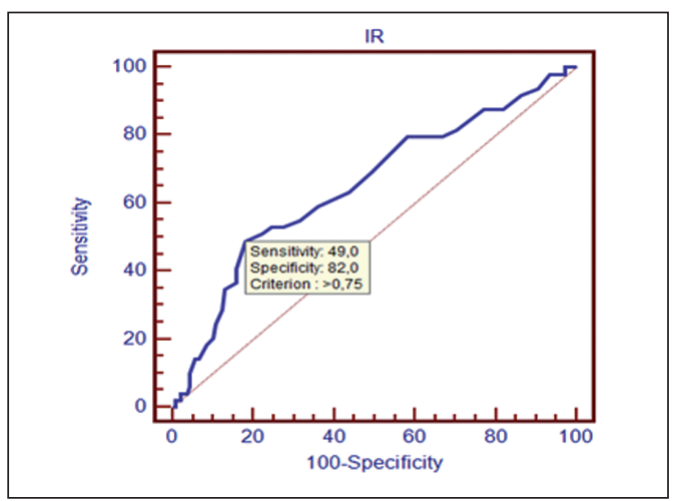

Figure 2.

The Kaplan-Mayer estimates graft survival correlated to $R I$ at 8 days $(p=0.0078)$.

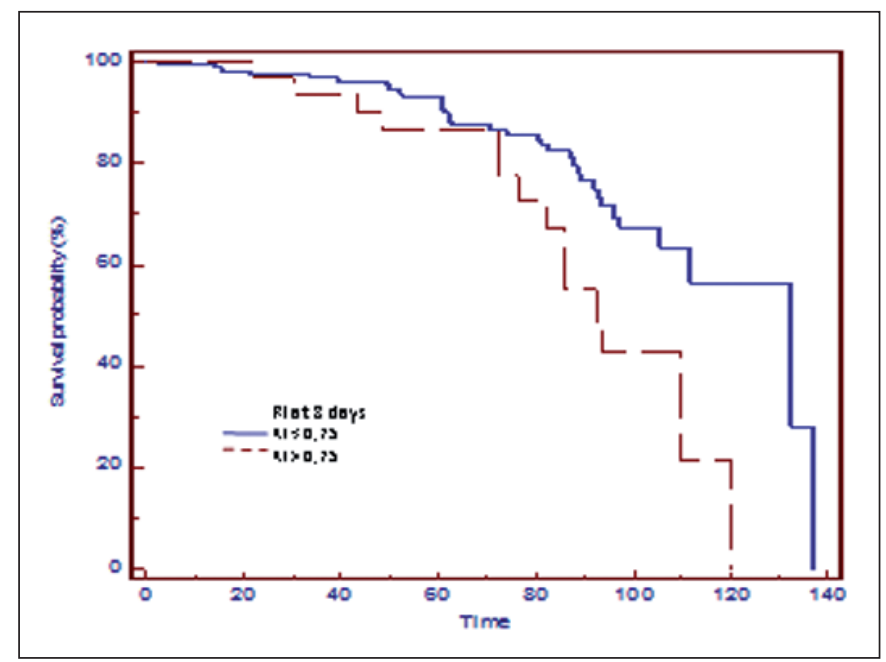

Figure 3.

The Kaplan-Mayer estimates graft survival correlated to $\mathrm{Rl}$ at 6 months $(p=0.02)$.

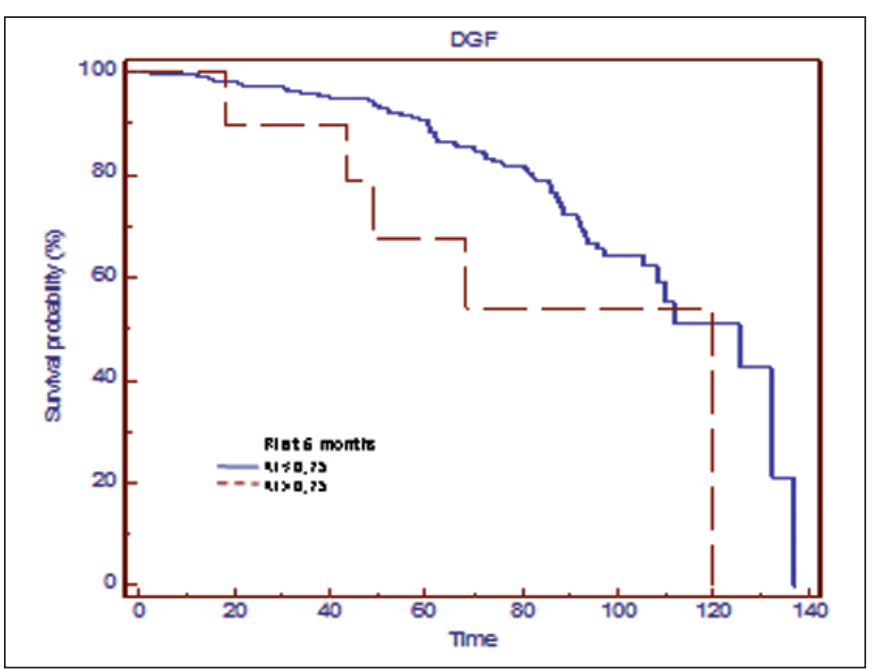


vs RI > 0.75: 31$)$, at 1 year $(\mathrm{RI} \leq 0,75: 229$ vs $\mathrm{RI}>0.75$ : 39 ) and at 3 years (RI $\leq 0,75: 224$ vs RI > 0.75: 44).

Kaplan-Maier estimates of cumulative GS we significantly worse in patients who had an RI $>0.75$, in correlation to the RI calculated at 8 days (Figure 2) and 6 moths (Figure 3 ) ( $p=0.0078$ and $p=0.02$ to 8 days and to 6 months). When the population was stratified in correlation to RI calculation at 1 years and at 3 years, its values is not correlated to the GS.

When we evaluated the OS in relationship to the RI, we did not find any relationship.

\section{Discussion}

After kidney transplantation, several complications may occur. For many years, research has been focused on non-invasive diagnostic techniques, that would be reliably predict the outcome of transplantation and graft function. Doppler ultrasonography is a useful tool for early evaluation of the kidney vasculature and function (9). According to previous studies, clinical parameters associated with increased RI were older donor and recipient age and vascular compliance (10-13).

Kolonko et al. concluded that the high RI values measured in the very early post-transplant period predict worse kidney graft function and increased risk of allcause graft loss, and he said the RI is not completely independent from the adverse influence of delayed graft function (DGF) on the premature graft loss (14). Other author concluded the same (7). Naesens et al. confirmed that the RI, routinely measured at predefined time points after transplantation, reflects characteristics of the recipient but not those of the graft (8).

We estimated that the RI has an importance when it is calculated in the early months after the transplantation. We identified a value of 0,75 as cut-off. The importance of RI is within 6 months, but it is correlated only with the GS but not with the OS.

\section{Conclusions}

The RI proved to be a good prognostic factor on survival organ when it was evaluated in the early months of followup after transplantation. This parameter does not appear, however, correlate with OS of the transplanted subject.

\section{ReFERENCES}

1. Vollmer WM, Wahl PW, Blagg CR. Survival with dialysis and transplantation in patients with end-stage renal disease. N EngJ Med. 1983; 308:1953-1958

2. Wolfe RA, Ashby VB, Milford EL, et al. Comparison of mortality in all patients on dialysis, patients on dialysis awaiting transplantation, and recipients of a first cadaveric transplant. N Engl J Med. 1999; 341:1725-1730,

3. Danavotich GM, ed. Handbook of kidney transplantation. 5th ed. Philadelphia: Lippincott Wiliams \& Wilkins, 2000.

4. Nankievell BJ, Kuypers DR. Diagnosis and prevention of chronic kidney allograft loss. Lancet. 2013; 378:1428-1437.

5. Pourcelot L. Velocimetrie ultrasonare Doppler. Seminare INSERM. Paris, France. Edition INSERM. 1974; 213-240.
6. Radermacher J, Mengel M, Ellis S, et al. The renal arterial resistance index and renal allograft survival. N Eng J Med. 2003; 349:115-124.

7. Impedovo SV, Martino P, Palazzo S, et al. Value of the resistive index in patient and graft survival after kidney transplant. Arch Ital Urol Androl. 2012; 84:279-282.

8. Naesens $M$, Heylen $L$, Lerut $E$, et al. Intrarenal resistive index after renal transplantation. 2013; 369:1797-1806.

9. Kahraman S, Genctoy G, Cil B, et al. Prediction of renal allograft function with early Doppler ultrasonography. Transplant Proc. 2004; 36:1348-1351.

10. Krumme B, Grotz W, Kirste G, et al. Determinants of intrarenal Doppler indices in stable renal allograft. J Am Soc Nephrol. 1997; 8:813.

11. Wang SM, Lai MK, Chueh SC, Chen J. The utility of resistance index of distal interlobular areteries evaluating renal graft function. Transplant Proc. 2004; 36:2184-2185.

12. Vallejos A, Alperovich G, Moreso F, et al. Resistive index and chronic allograft nephropathy evaluated in protocol biopsies as predictors of graft outcome. Nephrol Dial Transplant. 2005; 20:2511-2516.

13. Saracino A, Santarsia G, Latorraca A, Gaudiano V. Early assessment of renal resistance index after transplant can help predict longterm renal function. Nephrol Dial Transplant. 2006; 21:2916-2920.

14. Kolonko A, Chudek J, Zejda JE, Wiecek A. Impact of early kidney resistance index on kidney graft and patient survival during a 5-years follow-up. 2012; 27:1225-1231.

\author{
Correspondence \\ Saverio Forte, MD \\ saverio.forte@gmail.com \\ Pasquale Martino, MD \\ martino@urologia.uniba.it \\ Silvano Palazzo, MD \\ silvano.palazzo@alice.it \\ Matteo Matera, MD \\ materamatteo@libero.it \\ Floriana Giangrande, MD \\ giangrandefloriana@libero.it \\ Francesco Paolo Selvaggi, MD \\ selvaggi@urologia.uniba.it \\ Pasquale Ditonno, MD \\ ditonno@urologia.uniba.it \\ Michele Battaglia, MD \\ battaglia@urologia.uniba.it \\ Policlinico di Bari, Divisione Urologia Universitaria I \\ Piazza G. Cesare 11 - 70124 Bari, Italy
}

